\title{
FePt nanoparticles as a potential X-ray activated chemotherapy agent for HeLa cells
}

\author{
This article was published in the following Dove Press journal: \\ International Journal of Nanomedicine \\ 22 October 2015 \\ Number of times this article has been viewed
}

\author{
Yanhong Zheng' \\ Yunlan Tang ${ }^{2}$ \\ Zhirong Bao' \\ Hui Wang' \\ Feng Ren' \\ Mingxiong Guo² \\ Hong Quan' \\ Changzhong Jiang' \\ 'Key Laboratory of Artificial \\ Micro- and Nano-structures of the \\ Ministry of Education and Center \\ for Electronic Microscopy and \\ Department of Physics, Wuhan \\ University; ${ }^{2}$ College of Life Sciences, \\ Wuhan University, Wuhan, People's \\ Republic of China
}

\begin{abstract}
Nanomaterials have an advantage in "personalized" therapy, which is the ultimate goal of tumor treatment. In order to investigate the potential ability of FePt nanoparticles (NPs) in the diagnosis and chemoradiotherapy treatment of malignant tumors, superparamagnetic, monodispersed $\mathrm{FePt}(\sim 3 \mathrm{~nm})$ alloy NPs were synthesized, using cysteamine as a capping agent. The NPs were characterized by means of X-ray diffraction; transmission electron microscopy, Physical Property Measurement System, and Fourier transform infrared spectroscopy. The cytotoxicity of FePt NPs on Vero cells was assessed using an MTT assay, and tumor cell proliferation inhibited by individual FePt NPs and FePt NPs combined with X-ray beams were also collected using MTT assays; HeLa human cancer cell lines were used as in vitro models. Further confirmation of the combined effect of FePt NPs and X-rays was verified using HeLa cells, after which, the cellular uptake of FePt NPs was captured by transmission electron microscopy. The results indicated that the growth of HeLa cells was significantly inhibited by FePt NPs in a concentration-dependent manner, and the growth was significantly more inhibited by FePt NPs combined with a series of X-ray beam doses; the individual NPs did not display any remarkable cytotoxicity on Vero cells at a concentration $<250 \mu \mathrm{g} / \mathrm{mL}$. Meanwhile, the FePt NPs showed negative/positive contrast enhancement for MRI/CT molecule imaging at the end of the study. Therefore, the combined results implied that FePt NPs might potentially serve as a promising nanoprobe for the integration of tumor diagnosis and chemoradiotherapy.
\end{abstract}

Keywords: superparamagnetism, MRI/CT, chemoradiotherapy, intelligent nanoprobe

\section{Introduction}

Cancer is the world's foremost medical problem, with the number of new cases and cancerrelated deaths showing a disturbing increase. In 2012, the People's Republic of China was recorded as the country having the highest number of newly confirmed cancer cases. ${ }^{1}$ The past decade has seen improvements in the efficacy of tumor diagnosis and therapy, with treatment no longer limited to a single modality. ${ }^{2-6}$ While it has become more common to use dual-modal diagnosis and treatment, such as computed tomography/ magnetic resonance imaging (CT/MRI) with chemoradiotherapy and postsurgery radiotherapy, novel nanomaterials applied to medical science are regarded as promising agents for tumor diagnosis and therapy. Specifically, not merely nanocarriers used for drug delivery, but also noble metal and metal oxide nanoparticles (NPs) have revealed their potential in singlemodality tumor diagnosis and treatment. ${ }^{7-11}$ However, tumor treatment faces challenges such as imaging location and complications (radiodermatitis, radiation pneumonia, organ failure, etc). While traditional drug treatments and single-modality nanomaterials are unable to solve these problems, multi-modality agents may promote advancement and reduce adverse side effects of each therapeutic modality. Therefore, the study and exploration of multifunctional nanomaterials for combined various therapeutic modalities of tumor diagnosis and treatment is one of the most urgent affairs in medical research.
Correspondence:

Key Laboratory of Artificial Micro- and Nano-structures, Ministry of Education and Center for Electronic Microscopy and Department of Physics, Wuhan University, Wuhan 430072, People's Republic of China

Tel +86 27 68752567

Fax +862768753587

Email 00007962@whu.edu.cn;

czjiang@whu.edu.cn
International Journal of Nanomedicine 20I5:10 6435-6444

Dovepress

http://dx.doi.org/10.2147/IJN.S88458

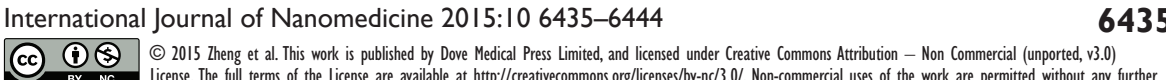
pemission from Dove Medical Press

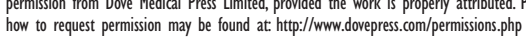


It is well known that there are three typical therapeutic methods for tumor diagnosis and treatment: chemotherapy, radiotherapy, and surgery. ${ }^{12}$ Although they are very useful methods for tumor therapy, they are still limited by several challenges and side effects. For example, surgery cannot always remove a tumor growing near vital organs, most agents used in chemotherapy are also toxic to normal organs and tissues, and radiotherapy cannot distinguish normal cells from tumor cells, thus resulting in complications. Naturally, combination therapy is increasing in popularity in clinical tumor treatment, as it combines the advantages of each of the various therapies, while avoiding the side effects. Nevertheless, the problem with combination therapy is that more drugs are used, and there are still rare agents can function throughout the whole process of combination therapy. Therefore, the research of multifunctional agents for combination therapy is very important and clinically significant. Specifically, the concurrent implementation of chemotherapy and radiotherapy, which is called chemoradiotherapy, has been regarded as an important therapy exemplification in the treatment modality for solid tumors over the past several decades. ${ }^{13-16}$ Compared with either chemotherapy, radiotherapy or sequential treatment, chemoradiotherapy has not only displayed the ability to consistently local tumor control, but also improve the curative ratio of cancer without surgical operation. ${ }^{13,17}$ In spite of the significant success that has been achieved, chemoradiotherapy is limited by its additional toxicity. Moreover, the prescription dose of radiation has not been reduced in chemoradiotherapy processes, which may damage normal tissues and organs. ${ }^{18}$ Therefore, there is an urgent need to improve the therapeutic gain factor of chemoradiotherapy. Promisingly, NPs possess several unique properties. Their excellent high X-ray absorption, control of drug release, enhancement of drug permeability, and retention effect leading to high NP concentration in tumor cells, make them strongly suitable for the improvement of chemoradiotherapy. Additionally, the magnetic properties of NPs could play important roles in tumor imaging contrast enhancement. ${ }^{19-21}$ Sun et al ${ }^{22}$ were the first to report the synthesis and characterization of monodisperse FePt NPs. Since then, FePt alloy NPs have attracted much attention due to their fascinating potential in the biomedical science field. ${ }^{23-26}$ For example, the abilities of NPs to inflict DNA damage in tumor cells, and catalyze oxygen reduction reaction make the NPs of great value in suppressing proliferation of tumor cells. ${ }^{27-29}$ Furthermore, compared to several commercial MRI/CT contrast agents, the superparamagnetic properties and X-ray absorption abilities of FePt NPs make them potential in vitro and in vivo dual-modality contrast agents (after surface engineering with functional molecules). ${ }^{30-33}$ Based on the extremely high $\mathrm{X}$-ray mass absorption coefficient of Pt, the hypothesis of the current study was that the NPs may increase the absorbed external beam dose in tumors, with decreased absorption of radiation in normal tissue and organs. Therefore, FePt NPs could be the precursor for CT, MRI, chemotherapy and radiotherapy all within one single agent, acting as a kind of multifunctional nanomedicine. ${ }^{34}$

In this work, the possibility of using superparamagnetic FePt alloy NPs as CT/MRI dual-modal imaging contrast agents and aforementioned chemoradiotherapy improvement is presented. FePt NPs were synthesized by using a chemical reduction method at a low temperature, and surface coating with cysteamine (Cys) to isolate the amine group for further target material conjugation. The characterizations of the NPs were performed by X-ray diffraction (XRD), transmission electron microscopy (TEM), Fourier transform infrared spectroscopy (FTIR), and comprehensive Physical Property Measurement System (PPMS). After the in vitro cytotoxicity of the NPs was evaluated using Vero cells, the growth inhibition effect of FePt NPs was evaluated by a 5-dimethylthiazol2-yl-2,5-diphenyl tetrazolium bromide (MTT) assay using HeLa cells with and without X-ray beam. Finally, the NPs' ability for medical imaging enhancement was measured by MRI/CT. The results indicated that the FePt NPs have potential as multimodality nanomedicine agents, in regard to the diagnosis and treatment of cancerous tumors.

\section{Materials and methods Materials}

Analytically pure chloroplatinic acid $\left(\mathrm{H}_{2} \mathrm{PtCl}_{6}\right)($ Reagent No 1 Factory Of Shanghai, Chemical Reagent Co., Ltd., Shanghai, People's Republic of China), 98\% iron acetylacetonate (Aladdin Industrial Corp. ${ }^{\circledR}$, CA, USA), Cys (Sigma-Aldrich Co., St Louis, MO, USA) were used in this research. Sodium borohydride (96\%), ethanol (analytically pure), anhydrous ethanol (analytically pure), ethylene glycol (analytically pure) were purchased from Sinopharm Chemical Reagent Co., Ltd, Shanghai, People's Republic of China.

\section{Synthesis of FePt NPs}

FePt NPs were prepared by using a chemical reduction method. Briefly, $1.5 \mathrm{~mL}$ oleylamine, $1.5 \mathrm{~mL}$ oleic acid, $0.1363 \mathrm{~g}$ iron acetylacetonate, and $100 \mathrm{~mL}$ anhydrous ethanol were mixed into three-necked round-bottomed flasks after 30 minutes of magnetic stirring. Twenty milliliters of $\mathrm{H}_{2} \mathrm{PtCl}_{6} \cdot 6 \mathrm{H}_{2} \mathrm{O}$-alcohol 
solution $(0.0193 \mathrm{mmol} / \mathrm{L})$ were transferred to the flask. Subsequently, $200 \mathrm{~mL}$ sodium borohydride-alcohol solution $(0.0657 \mathrm{mmol} / \mathrm{L})$ was gradually added after 30 minutes of magnetic stirring with argon flow. The mixture was heated to $40^{\circ} \mathrm{C}$ and stirred with argon flow for 1 hour. The black product was precipitated out by centrifugation $(10,000 \mathrm{rpm}$ for 6 minutes), and then dried in a vacuum for 48 hours at $40^{\circ} \mathrm{C}$, after which, FePt NPs were obtained.

\section{Ligand exchange}

Dry FePt NPs $(10 \mathrm{mg})$ were dissolved in ethyl alcohol by sonication. Cys $(\sim 100 \mathrm{mg})$ was added into this solution and dissolved by sonication at $30^{\circ} \mathrm{C}$. This mixture was sonicated for 6 hours at $40^{\circ} \mathrm{C}$. The unconnected ligand in solution was then washed away by ethyl alcohol. Finally, the surfacecoated FePt NPs were collected, and stored in a bottle filled with $\mathrm{N}_{2}$.

\section{FePt NPs characterization}

The formation and the phase structures of the samples were identified by powder XRD on a Bruker D8/Advance-X ray Diffractometer (Bruker Corporation, Billerica, MA, USA), using $\mathrm{Cu} \mathrm{K} \alpha$ radiation $(\lambda=1.5406 \AA$ ). The morphology, the phase structure, and the degree of dispersion of the samples were observed on an EM2010FEF- $\Omega$ transmission electron microscope (JEOL, Tokyo, Japan). FTIR spectra were recorded and the ligand attachment was verified by the Nicolet 5700 FTIR Spectrometer. The magnetic properties of the samples were measured by PPMS (Quantum Design, Inc., San Diego, CA, USA). The measurements between $-10,000$ and $10,000 \mathrm{Oe}$ at $300 \mathrm{~K}$ were chosen.

\section{Cell culture and treatment}

The Vero cell line and HeLa cell line (both purchased from the Type Culture Collection of the Chinese Academy of Sciences, Shanghai, People's Republic of China) were both maintained in Minimum Essential Medium (Thermo Fisher Scientific, Waltham, MA, USA) which contained $10 \%$ fetal bovine serum (Thermo Fisher Scientific, Waltham, MA, USA), 100 units penicillin $\mathrm{mL}^{-1}$, and $100 \mu \mathrm{g}$ streptomycin $\mathrm{mL}^{-1}$ (Beyotime Biotechnology, Shanghai, People's Republic of China) in a $37^{\circ} \mathrm{C}$ incubator (Thermo Fisher Scientific Waltham, MA, USA) with $5 \% \mathrm{CO}_{2}$.

\section{Cytotoxicity assay on Vero cells}

Vero cells were seeded in 96-well plates for 24 hours, with $1.2 \times 10^{4}$ cells in each well. A series of FePt-Cys NPs were dispersed in the cells, with final concentrations of 12.5, 25,
125 , and $250 \mu \mathrm{g} / \mathrm{mL}$ for an additional day. Survival fraction was measured by the MTT assay. After all the cells were treated, $20 \mu \mathrm{L}$ MTT $(20 \mathrm{mg} / \mathrm{L})$ were placed into each well for 4 hours at $37^{\circ} \mathrm{C}$. Finally, $150 \mu \mathrm{L}$ dimethyl sulfoxide (DMSO) was added to each well, and the 96-well plates were shaken for 10 minutes. A microplate reader (Thermo Fisher Scientific Waltham, MA, USA) was used to determine the absorbance values at $590 \mathrm{~nm}$.

\section{Combined FePt treatment and external beam radiation dose response on HeLa cells}

The HeLa cell line was cultured in 96-well plates, with $2.5 \times 10^{3}$ cells in each well. After 24 hours, a series of FePtCys NPs were added into each well, with final concentrations of $0,25,50$, and $100 \mu \mathrm{g} / \mathrm{mL}$. Two hours later, the groups of HeLa cells were irradiated with 0, 2, 4, 6, 8, and 10 Gy 6 MV photon beams, respectively, using the Varian Clinac ${ }^{\circledR}$ iX Linear Accelerator (Varian Medical Systems, Palo Alto, CA, USA) at the Zhongnan Hospital of Wuhan University, Wuhan, Hubei, People's Republic of China. Following incubation of the cells at $37^{\circ} \mathrm{C}$ for 72 hours, an MTT assay was used to measure the cell viability. The two experiment groups were then prepared for measurement of their interactive effect by injecting $0,12.5,25$, and $50 \mu \mathrm{g} / \mathrm{mL} \mathrm{FePt-Cys}$ NPs before and after 2 Gy X-ray beam irradiation. After the cells were incubated for 72 hours, the MTT assay was used to collect the data. All data were expressed as mean \pm standard deviation, and a two-way analysis of variance (ANOVA) and a Student's $t$-test.

\section{Cellular uptake}

HeLa cells were incubated with $25 \mu \mathrm{g} / \mathrm{mL}$ FePt-Cys NPs for 24 hours. After the medium had been removed, 2.5\% glutaraldehyde and $1 \%$ osmic acid were added to fix the cells. Subsequently, the dehydration of cells was performed by the serial addition of ethanol $(30 \%, 50 \%, 60 \%, 70 \%$, $80 \%$, and $90 \%$ concentrations), and the embedding of cells was achieved using EPON812. The cells were then polymerized in an oven at $60^{\circ} \mathrm{C}$ for 24 hours. The cells were sliced ( $\sim 100 \mathrm{~nm}$ thick) using a diamond knife on a Leica ultramicrotome (Leica Microsystems, Wertzlar, Germany), and placed onto a copper grid. Images were captured by TEM.

\section{$M R I$ and $C T$ imaging in vitro}

A column of centrifuge tubes which contained 0.01-100 $\mathrm{mmol} / \mathrm{L} \mathrm{FePt} \mathrm{NPs} \mathrm{and} \mathrm{a} \mathrm{phosphate} \mathrm{buffer} \mathrm{saline} \mathrm{(PBS)} \mathrm{control}$ 
was used for MRI and CT contrast imaging. $\mathrm{T}_{2}$-weighted three-dimensional spin echo sequences for in vitro MRI (MAGNETOM Trio Tim 3.0T, Siemens, Munich, Bavaria, Germany) were performed at the Hospital of Wuhan University, Wuhan, Hubei, People's Republic of China, and used the following parameters: a repetition time of 550 milliseconds, an echo time of 15 milliseconds, a flip angle of $15^{\circ}$, a slice thickness of $1.5 \mathrm{~mm}$, and a matrix of $256 \times 196$ pixels. The CT imaging (Sensation Cardiac 64x, Siemens, Munich, Bavaria, Germany) parameters were as follows: slice thickness, $3 \mathrm{~mm}$; $120 \mathrm{kvp}, 80 \mathrm{~mA}$; filed, $512 \times 512$; gantry rotation time, 0.3 second; table speed, $30 \mathrm{~mm} /$ rotation.

\section{Results}

\section{Characterization of FePt NPs}

Figure 1A shows the XRD patterns of the FePt NPs. Compared to the powder diffraction file standard card, all four characteristic peaks of the chemically-disordered, face-centered cubic structure of FePt were clearly identified in the curve, which had planes of (111), (200), (220), and (311), respectively. ${ }^{27,28}$ No other characteristic peak of iron oxide or pure Pt was found in the powder XRD. Interestingly, as the values calculated from broader XRD by the Debye-Scherrer formula usually suggests smaller sizes, the XRD scan was in line with the size of FePt NPs calculated from the TEM images. ${ }^{35}$ The TEM images of FePt NPs are shown in Figure 1B. The average size of FePt NPs was $3.11 \pm 0.53 \mathrm{~nm}$. This size was calculated by averaging the sizes of 100 NPs; size distribution is shown in Figure 1C. The FePt NPs appeared to have globoseshaped nuclei (ultimately determined to be cuboctahedral when visualized with high-resolution TEM). ${ }^{25}$ The TEM images showed no agglomeration, which suggests the excellent monodispersity of FePt NPs because of two reasons: 1$)$ a low reaction temperature $\left(40^{\circ} \mathrm{C}\right)$, and 2$)$ the negatively-charged surface resulting in the interparticle repulsive force. ${ }^{35}$ Figure $1 \mathrm{D}$ shows that the saturated mass magnetization of FePt NPs was $1.13 \mathrm{emu} / \mathrm{g}$, coupled with the absence of coercive force, which indicated that the FePt NPs exhibited superparamagnetic behavior at $300 \mathrm{~K} .{ }^{36}$ The ligand exchange was confirmed by FTIR measurements in the range of $400-4,000 \mathrm{~cm}^{-1}$ with $2 \mathrm{~cm}^{-1}$ resolution at room temperature. The recorded spectrums of bare FePt NPs, Cys, and Cys-capped FePt NPs are shown in Figure 1E. The absorption peak of $\sim 3,425 \mathrm{~cm}^{-1}$ was assigned to the $\mathrm{N}-\mathrm{H}$ stretching vibration. The vibration of the $\mathrm{C}-\mathrm{H}$ bond corresponded to the absorption peak at $\sim 3,025 \mathrm{~cm}^{-1}$. The $\mathrm{S}-\mathrm{H}$ bond vibration absorption peak at $2540 \mathrm{~cm}^{-1}$ disappeared on the spectrum of FePt-Cys, and at the fingerprint region (1300-900 $\mathrm{cm}^{-1}$ ) the FePt-Cys spectrum shows many different from the other two; this suggests that the Cys molecules were connected to the surface of FePt NPs by the coordinate bond between $\mathrm{S}$ and $\mathrm{Pt}$ atoms, which have high affinity for each other. ${ }^{37}$

\section{Cytotoxicity assay on Vero cells}

The in vitro cytotoxicity of Cys-capped FePt NPs was evaluated in the Vero cell line using the MTT assay with a series of different concentrations. The cell viability after incubation for 24 hours is shown in Figure 2. The original data of concentrations $12.5,25$, and $125 \mu \mathrm{g} / \mathrm{mL}$ FePt-Cys NPs did not show a significant difference when compared to the control group of the Vero cells, and this is reflected in the cell viability (almost 100\%) illustrated in Figure 2. When the concentration of FePt NPs was raised to $250 \mu \mathrm{g} / \mathrm{mL}$, the Vero cells showed only a $\sim 14 \%$ loss of cell viability. These results indicate that FePt NPs have no significant cytotoxicity on Vero cells in vitro at concentrations below $250 \mu \mathrm{g} / \mathrm{mL}$.

\section{Combined FePt therapy and external beam radiation dose response on HeLa cells}

In order to assess the ability of FePt-Cys NPs with or without X-ray beams on cell inhibition of HeLa cells, HeLa cells were cultured with FePt-Cys NPs irradiated by serial doses of X-ray beams. As shown by the data displayed in Figure 3, the survival rates of HeLa cells were significantly suppressed by FePt-Cys NPs without irradiation, in a concentration-dependent manner, in the range of $25-100 \mu \mathrm{g} / \mathrm{mL}$. For example, the cell viability of the group treated with $25 \mu \mathrm{g} / \mathrm{mL}$ FePt-Cys NPs and irradiated by 2 Gy X-ray beams showed no significant difference to that of the group treated with $100 \mu \mathrm{g} / \mathrm{mL}$ FePt-Cys NPs only; this was slightly lower than that of the groups irradiated by 8 Gy or 10 Gy X-Ray beams. Therefore, the results suggest that FePt-Cys NPs can not only inhibit the growth of HeLa cells in a concentration-dependent manner, but also show a combination effect with X-rays to suppress the proliferation of HeLa cells more remarkably. By comparison, HeLa cells seemed to be more sensitive to the NPs than Vero cells.

To further confirm the existence of the potential interaction effects between FePt-Cys NPs and X-ray, FePt-Cys NPs were injected into the wells cultured with HeLa cells pre or postirradiation, and all the experiment groups were irradiated by the 2 Gy X-ray beam. As shown in Figure 4, cell viabilities of the group that only received irradiation 

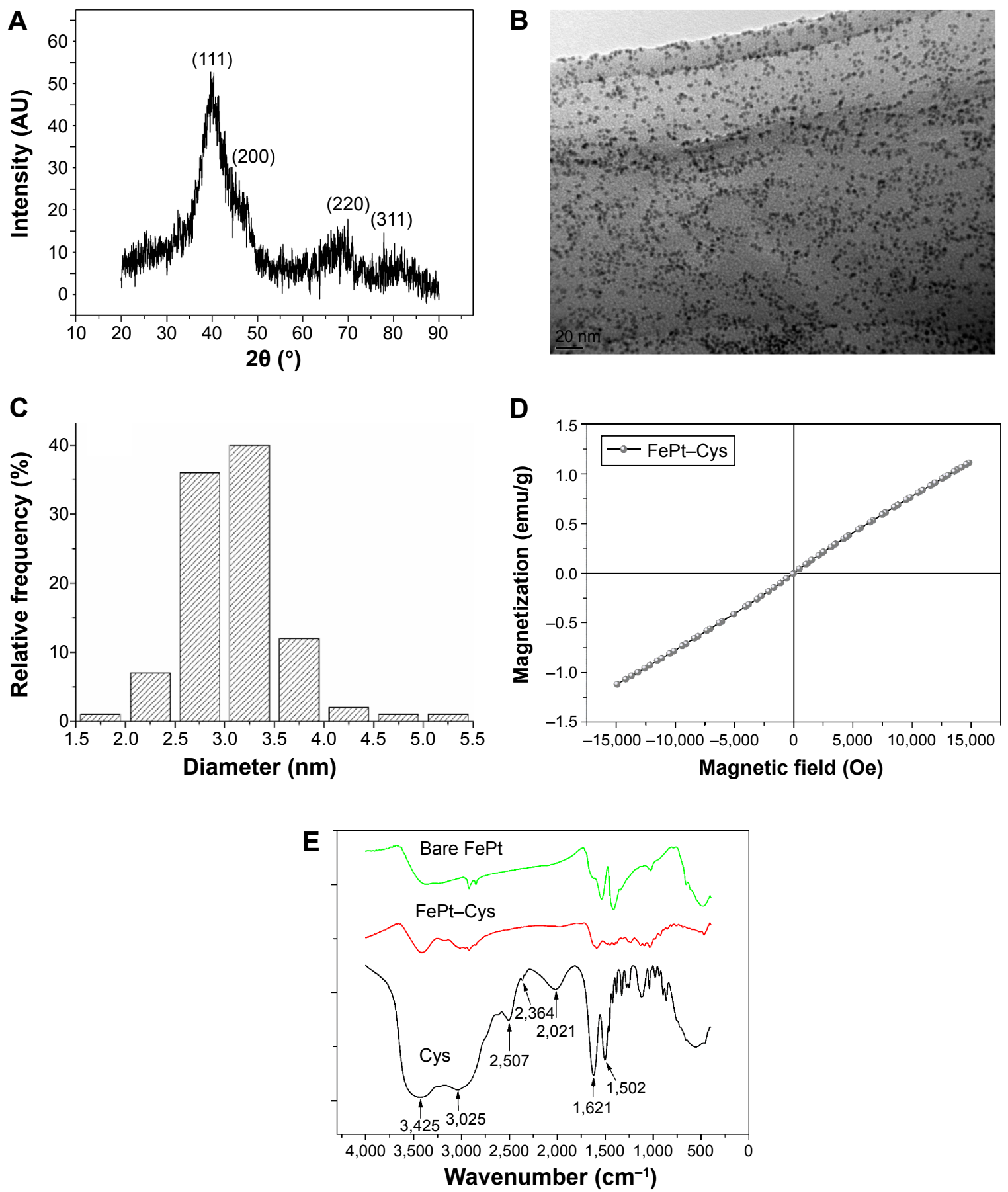

Figure I The XRD scans of FePt NPs (A), the TEM image of FePt NPs (B), the size distribution of FePt NPs randomly acquired from 100 NPs (C), the hysteresis loop of FePt NPs recorded between -15,000 and 15,000 Oe at $300 \mathrm{~K}$ (D), and the FTIR spectrum of Bare FePt NPs, FePt-Cys NPs and Cys (E).

Abbreviations: XRD, X-ray diffraction; NPs, nanoparticles; TEM, transmission electron microscopy; AU, arbitrary unit.

remained consistent. Once NPs were injected at 2 hours pre- or postirradiation, cell viabilities showed $\sim 5 \%, \sim 9 \%$, and $\sim 10 \%$ differences between the two kinds of group at $12.5,25$, and $50 \mu \mathrm{g} / \mathrm{mL}$ concentrations of the FePt-Cys NPs, respectively. Therefore, all the cellular results demonstrate that FePt-Cys NPs not only remarkably inhibited the cell proliferation of HeLa cells, but also had combination effects with X-ray beams to suppress the proliferation of HeLa cells.

\section{Cellular uptake}

The cellular uptake of FePt NPs by HeLa cells was captured by TEM. As shown in Figure 5, the vesicles containing black FePt NPs were observed, proving the direct cellular 


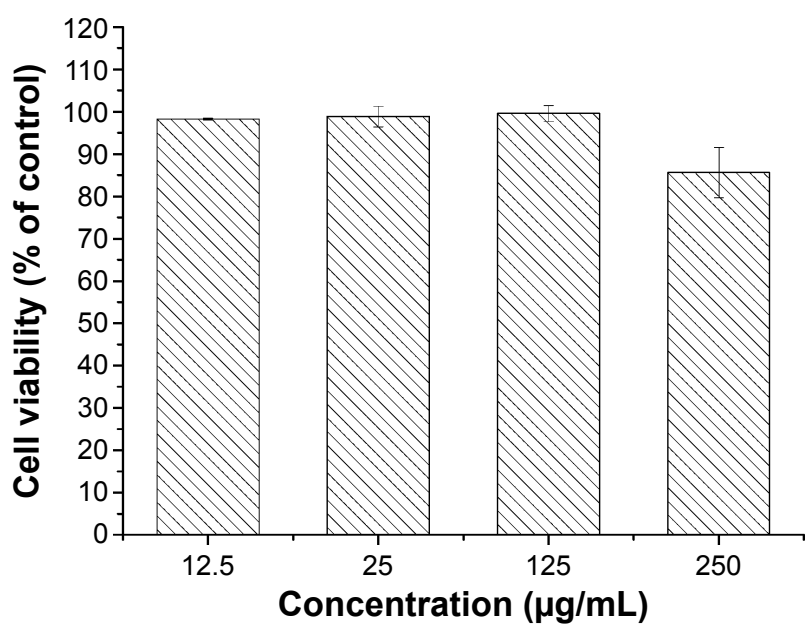

Figure 2 Cell viability of Vero cells treated with FePt NPs. Note: Incubation time was 24 hours.

Abbreviation: NPs, nanoparticles.

internalization of the FePt NPs. The left side of Figure 5A shows that HeLa cells, the initial phase, and during the formation of the vesicles absorbed the black FePt NPs. The release and diffusion of the vesicle-wrapped FePt NPs into the cytoplasm are shown in the right side of Figure 5A. As can be seen at the bottom of Figure 5C, the FePt NPs were penetrating the membrane of cellular nuclei. The right sides of Figure 5A and D shows that the pathway of NPs is a kind of endocytosis called macropinocytosis.

\section{$M R I$ and $C T$ image in vitro}

The in vitro $\mathrm{T}_{2}$-weighted MRI images of various FePt concentrations were captured by a $3 \mathrm{~T}$ MR imager, and the calculated intensity of each tube compared to that

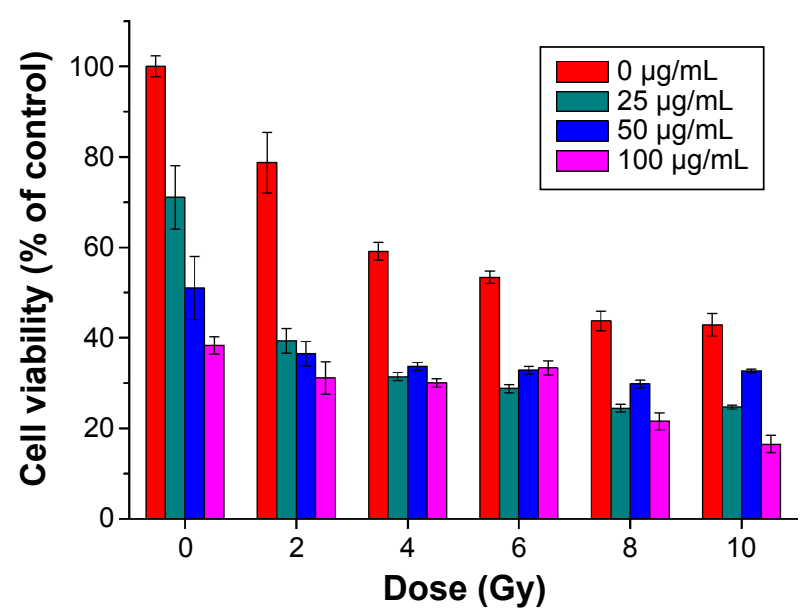

Figure 3 The growth inhibition of HeLa cells treated with FePt NPs combined with $X$-ray beams.

Note: Incubation time was 72 hours.

Abbreviation: NPs, nanoparticles.

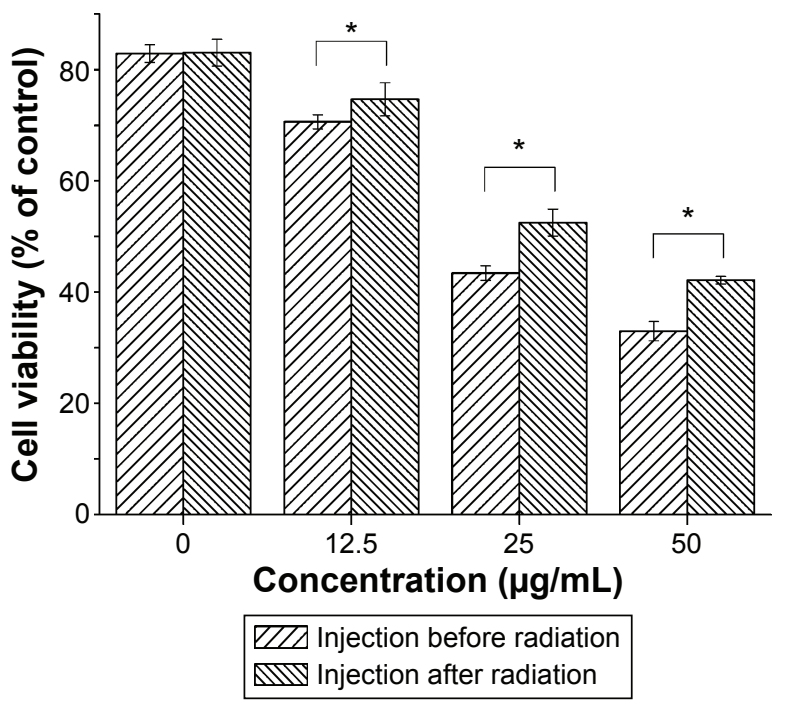

Figure 4 The growth inhibition of HeLa cells treated with FePt NPs before and after 2 Gy X-ray irradiation.

Notes: Incubation time was 72 hours; $* P<0.05$.

Abbreviation: NPs, nanoparticles.

of the PBS tube was normalized, as shown in Figure 6. The $100 \mathrm{mM} \mathrm{FePt}$ tube presented the most effective negative MR contrast enhancement, with a signal intensity decrease of $\sim 40 \%$. Overall, as shown by the MRI, FePt NPs demonstrated an excellent inverse contrast in vitro in a concentration-dependent manner. The in vitro $\mathrm{CT}$ imaging was examined at various FePt concentrations, and the positive contrast effects of the NPs were certified by these results. The calculated $\mathrm{CT}$ value of each tube was pointed and plotted, and shown in Figure 6B, with the CT value of PBS set to 0 . In accordance with the results of the MRI, the $100 \mathrm{mM}$ FePt tube exerted the most effective positive CT contrast enhancement. Overall, the FePt NPs displayed contrast enhancement in the $\mathrm{CT}$ in vitro in a concentrationdependent manner.

\section{Discussion}

To date, it has been reported that FePt alloy NPs significantly inhibit the growth of tumor cells (such as U87, H4, HeLa, A2780, A431, Sk-Br3, and HEK-293) by acting as yolk in a yolk-shell nanocrystal, coated with phospholipid or other chemical compounds..$^{35,38-41}$ Furthermore, in the area of medical imaging, FePt NPs are considered to have great potential in MRI and MRI/CT dual-model imaging as a regenerative medicine. ${ }^{31,33}$ However, we confirm that there is still a paucity of research on FePt NPs in the radiotherapy field, in which the NPs may hold potential. The current study reports the synthesis of $3.11 \pm 0.53 \mathrm{~nm}$ monodispersed FePt NPs by a chemical reduction method, and the coating of these NPs 

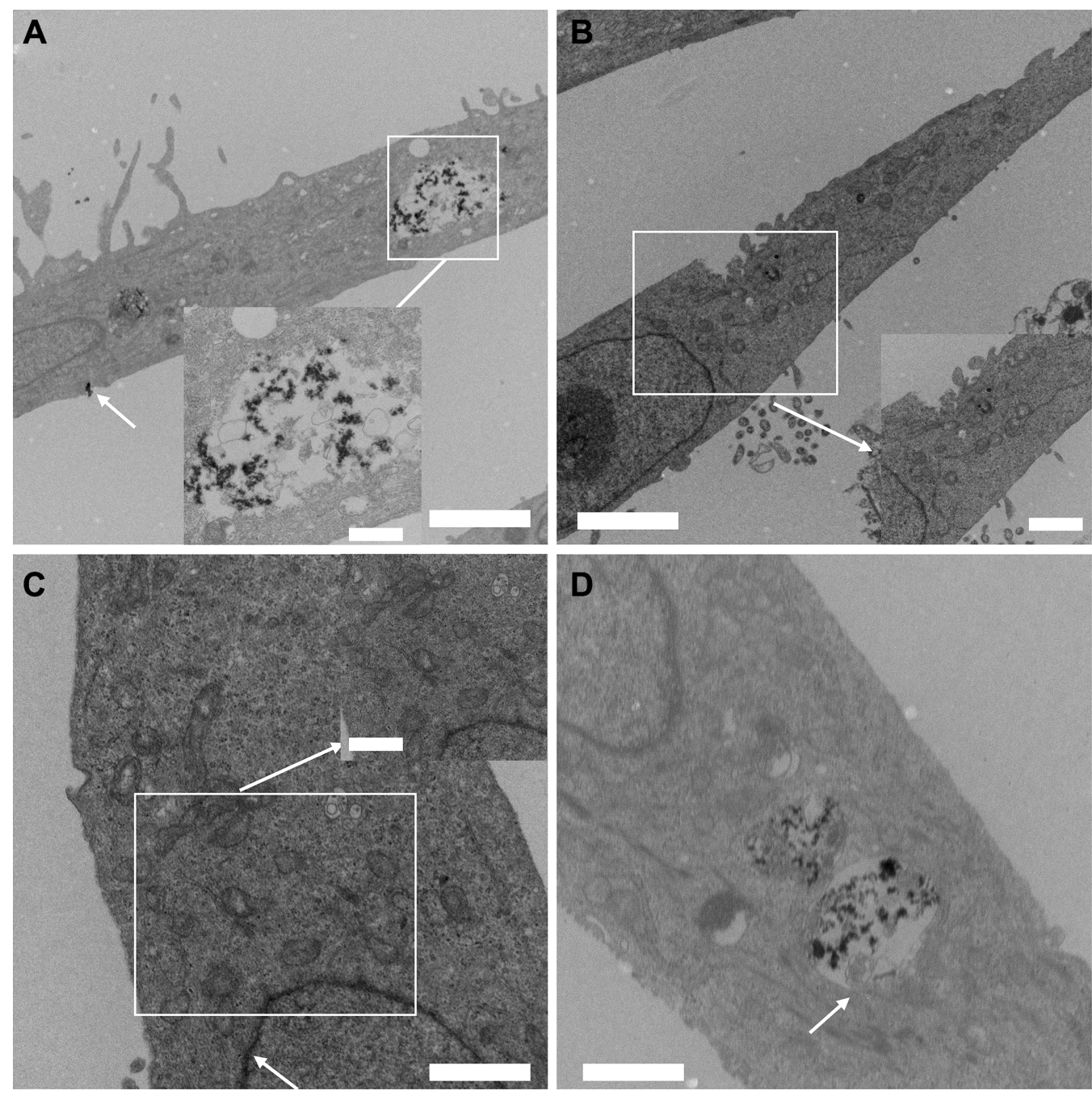

Figure 5 The TEM images of cellular uptake by FePt NPs.

Notes: The arrows in are used to point out FePt NPs entering the cell in $\mathbf{A}$, and he FePt NPs entering the cell nucleus membrane in $\mathbf{C}$ and finally, the FePt NPs enwrapped by the vesicles in $\mathbf{B}$ and $\mathbf{D}$. The scale of each TEM image is displayed in the lower right corner: $2 \mu \mathrm{m}$ and $500 \mathrm{~nm}(\mathbf{A}) ; 2 \mu \mathrm{m}$ and I $\mu \mathrm{m}(\mathbf{B}) ; \mathrm{I} \mu \mathrm{m}$ and $500 \mathrm{~nm}(\mathbf{C})$; and I $\mu \mathrm{m}(\mathbf{D})$. Abbreviations: TEM, transmission electron microscopy; NPs, nanoparticles.

with Cys. The potential applications of NPs in chemoradiotherapy and medical imaging contrast enhancement were studied in HeLa cells exposed to a $6 \mathrm{MV}$ photon beam, and by MRI/CT imaging system in vitro, respectively. The results proved that FePt NPs inhibited the growth of HeLa cells in a concentration-dependent manner, and when the NPs and X-ray beams were combined, the growth inhibition of HeLa cells varied remarkably compared to that treated by NPs or X-rays alone. The results further confirmed the existence of the potential interactive effect between FePt NPs and X-ray beams, and demonstrated the positive and negative imaging contrast enhancement of $\mathrm{CT}$ and MRI by FePt NPs. Compared to cisplatin, a kind of traditional anticancer platinum complex, FePt NPs could damage HeLa cells alternatively between HeLa and Vero cells, due to the acid-based environment intracellular. Therefore, collectively, these results could be the definitive backup to endorse FePt NPs as potential novel nanoprobes for the integration of diagnosis and chemoradiotherapy on HeLa cells. The results displayed in Figures 2 and 3 indicate that the FePt NPs show significant cytotoxicity on HeLa cells, while not significantly suppressing the proliferation of Vero cells at a concentration below $250 \mu \mathrm{g} / \mathrm{mL}$. Previously, Xu et a ${ }^{38}$ suggested that FePt NPs showed the controlled release in a low $\mathrm{pH}$ solution, while there was little degradation of FePt NPs in a solution of $\mathrm{pH}$ 7.4. Gao et $\mathrm{al}^{29}$ indicated that, in HeLa cells, the FePt cores of FePt@ $\mathrm{CoS}_{2}$ were oxidized and degraded in secondary lysosome with a low-pH environment. Therefore, the current study speculates that the low $\mathrm{pH}$ environment may promote the degradation of FePt 

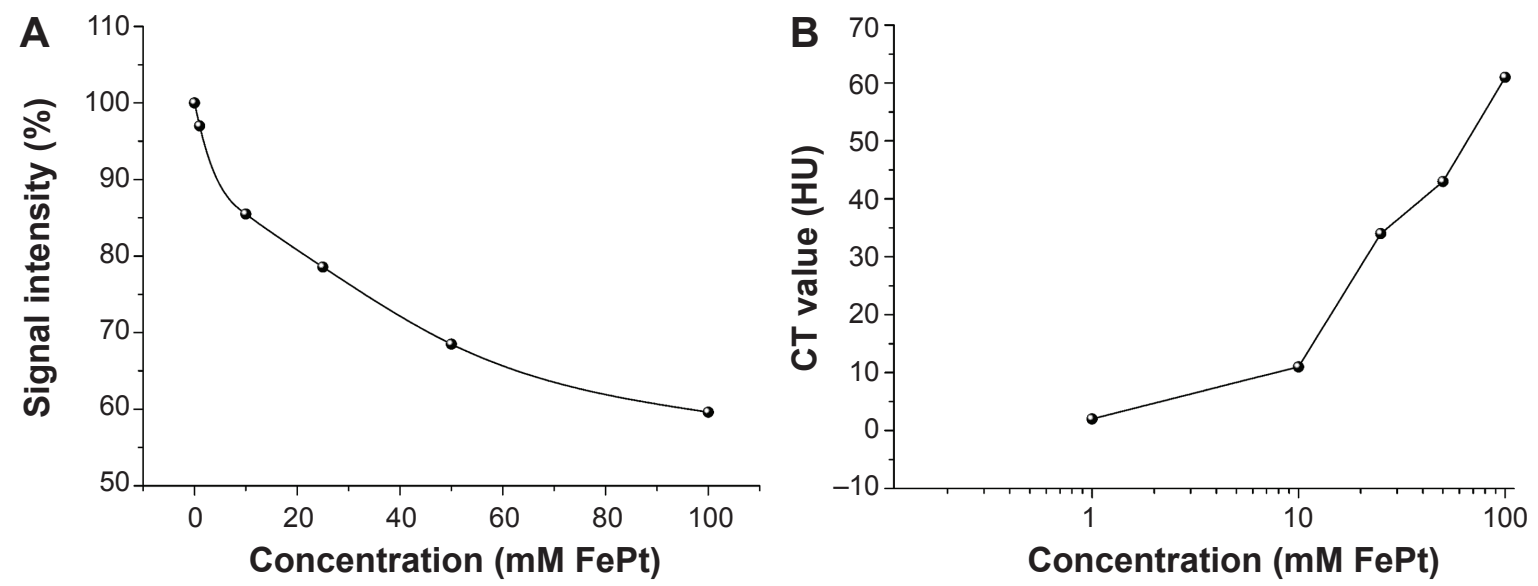

Figure 6 The calculated data curve of MRI/CT molecule imaging contrast enhancement with FePt NPs. Notes: (A) MRI negative contrast enhancement; (B) CT positive contrast enhancement.

Abbreviations: NPs, nanoparticles; MRI, magnetic resonance imaging; CT, computed tomography.

NPs, triggering the release of ions to damage tumor cells, either directly or indirectly, while few free ions are released in normal cells. To achieve a more effective specific suppression, further studies of engineering the surface of $\mathrm{FePt}$ NPs are needed, and are currently in progress. As shown in Figures 3 and 4, FePt NPs inhibited the growth of HeLa in a concentration-dependent manner without irradiation, and the suppression ratios remarkably increased when FePt NPs were combined with irradiation. These results suggested that the NPs not only acted as a chemosensitization agent, but also performed as a radiosensitizer under the environment of irradiation. What was of most interest in the current study was that the low concentration of FePt NPs combined with low-dose X-ray exposure suppressed the proliferation of HeLa cells more significantly than the separate high-dose X-ray beaming on HeLa cells (Figure 3). In other words, the utilization of FePt NPs in chemoradiotherapy may sensitize tumor cells on the one hand, and reduce the required radiation dose on the other. Meanwhile, the FePt NPs showed little cytotoxicity on Vero cells at low concentration. Therefore, the results suggested that FePt NPs may potentially increase the therapeutic gain factor through radiosensitizing, chemosensitization, and reducing the dose absorbed by normal cells. According to the above discussion, the as-synthesized FePt NPs may play a role in the chemoradiotherapy in vitro model as simple but efficient nanoprobes. Their mechanism of cellular action manifested itself in several ways. ${ }^{10,42,43}$ First, as the FePt NPs were superparamagnetic and water-soluble after surface capping with Cys, the signal intensity of MRI/CT images were negatively/positively enhanced in a concentration-dependent manner (Figure 6). This phenomenon may help to improve the accuracy of tumor location, especially helpful for the location of isolated tumor cells, which have already infiltrated normal tissues and may be the important cause of tumor recurrence. Second, the results showed that FePt NPs suppressed the proliferation of HeLa cells in a concentration-dependent manner as a chemotherapy agent. In order to investigate the damage mechanism caused by FePt NPs, the cellular uptake of FePt NPs was observed by TEM (Figure 5). The NPs surrounding tumor cells were endocytosed by the vesicles, demonstrating a nonspecific endocytic process called macropinocytosis. ${ }^{44,45}$ Macropinocytosis involves the internalization of large areas of the plasma membrane together with large amounts of fluid. After invagination of membrane plasma and then suspension within small vesicles, these pinocytosis vesicles containing FePt NPs subsequently fuse with lysosomes. Then the chemically stable FePt NPs partially degrade (hydrolyze) into highly active $\mathrm{Pt}^{2+}, \mathrm{Fe}^{2+}$, and $\mathrm{Fe}^{3+}$ ions in acid environments $\left(\mathrm{pH} \approx 4.8\right.$, mainly in lysosome). The released $\mathrm{Pt}^{2+}$ ions from primary or secondary lysosomes may then enter into the nuclei and mitochondria, coordinate with 5'-GG-3' bases of DNA, prevent DNA from replicating properly, and lead to the apoptosis of tumor cells. ${ }^{46,47}$ Alternatively, the released $\mathrm{Fe}^{2+}$ and $\mathrm{Fe}^{3+}$ ions may trigger an excess of highly reactive oxygen species (ROS) levels as Fenton-like catalysts. The ROS, such as the hydroxyl radical $(\bullet \mathrm{OH})$ and carboxyl radical $(\bullet \mathrm{OOH})$, are powerful agents for damaging various matter in cells, leading to cell death. ${ }^{27,38}$ Therefore, FePt NPs may have potential to act as chemotherapy agents to kill tumor cells. As demonstrated in the current study, FePt NPs combined with X-ray beams suppressed the proliferation of HeLa cells more efficiently as a radiosensitizer (Figure 4). The mechanism of this phenomenon may be 
attributed to the partial pressure increase of oxygen which, when accompanied by the excess generation of ROS, led to the increase of HeLa cells' radiosensitivity at the moment of irradiation. ${ }^{48}$ Because of the excellent X-ray mass absorption coefficient of the $\mathrm{Pt}$ atom, ${ }^{49}$ the original divergence and homogeneous nature of the X-ray beam could be focused on HeLa cells, aiming to concentrate energy to damage HeLa cells precisely. Here, FePt NPs played a part as radiosensitizers in the progress of a tumor radiotherapy model. Moreover, it would not be farfetched to surmise that normal tissues and organs surrounding tumor cells would absorb less energy from FePt NP-mediated radiotherapy treatment. Indeed, this factor increases the therapeutic gain of FePt NPs. In addition, the increased precision of this method may further promote the degradation of FePt NPs, a hypothesis which is currently being explored. In a nutshell, the current study has proposed that the intelligent application of FePt alloy NPs may improve cancer therapy and imaging effects and, as a multifunctional nanoprobe, decrease the occurrence of complications in the integration of tumor diagnosis and therapy. This idea could satisfy the ultimate goal of personalized chemoradiotherapy. Future work will focus upon the FePt surface engineering of targeted molecules, as well as further exploration of the interactive effect of FePt NPs and X-rays upon the damage of tumor cells, while simultaneously protecting normal cells.

\section{Conclusion}

The current study reports the synthesis of superparamagnetic, monodispersed FePt NPs with ligand exchange mediated by Cys. Cytotoxicity was assessed in Vero cells, and the potential utilization of the FePt NPs for integration of chemotherapy and radiotherapy was evaluated in HeLa cells in vitro. Finally, the prospective application of FePt NPs as dual-model contrast agents for MRI/CT molecular imaging was measured via MRI and CT scans in vitro. The results suggested that FePt NPs inhibited the growth of HeLa cells in a concentration-dependent manner while avoiding the effects of significant toxicity to Vero cells at concentrations below $250 \mu \mathrm{g} / \mathrm{mL}$. The proliferation of HeLa cells was remarkably suppressed by FePt NPs with serial doses of X-ray beams, which showed a greater efficacy than the same concentrations of individual FePt NPs do. The combined effect of FePt NPs and X-ray beam was verified in HeLa cells. Altogether, these results indicated that FePt NPs are a promising nanoprobe for the integration of diagnosis and chemoradiotherapy for tumor treatment.

\section{Acknowledgments}

This study was financially supported by the Natural Science Foundation of China (No 10875092 and 31271511), and the Natural Science Foundation of Hubei Province of China (No 2012KB04449). The authors are grateful to the Department of Radio- and Chemo-therapy, Zhongnan Hospital of Wuhan University, Wuhan, People's Republic of China, and Elekta Instrument (Shanghai) Ltd. for their assistance with the project.

\section{Disclosure}

The authors report no conflicts of interest in this work.

\section{References}

1. Bernard S, Christopher PW. World cancer report 2014. International Agency for Research on Cancer. Lyon: IARC Press; 2014.

2. Clarke M, Collins R, Darby S, et al. Effects of radiotherapy and of differences in the extent of surgery for early breast cancer on local recurrence and 15-year survival: an overview of the randomised trials. Lancet. 2005;366(9503):2087-2106.

3. Early Breast Cancer Trialists' Collaborative Group. Effect of radiotherapy after breast-conserving surgery on 10 -year recurrence and 15 -year breast cancer death: meta-analysis of individual patient data for 10,801 women in 17 randomised trials. Lancet. 2011;378(9804):1707-1716.

4. Rebecca WO, Richard MA. Combined chemotherapy and radiotherapy (without surgery) compared with radiotherapy alone in localized carcinoma of the esophagus. Cochrane Database Syst Rev. 2003;(1):CD002092.

5. Kocher M, Soffietti R, Abacioglu U, et al. Adjuvant whole-brain radiotherapy versus observation after radiosurgery or surgical resection of one to three cerebral metastases: results of the EORTC 22952-26001 study. J Clin Oncol. 2011;29(2):134-141.

6. James ND, Hussain SA, Hall E, et al. Radiotherapy with or without chemotherapy in muscle-invasive bladder cancer. New Engl J Med. 2012;366(16):1477-1488.

7. Brannon-Peppas L, Blanchette JO. Nanoparticle and targeted systems for cancer therapy. Adv Drug Deliv Rev. 2012;64:206-212.

8. Peer D, Karp JM, Hong S, Farokhzad OC, Margalit R, Langer R. Nanocarriers as an emerging platform for cancer therapy. Nat Nanotechnol. 2007;2(12):751-760.

9. Riehemann K, Schneider SW, Luger TA, Godin B, Ferrari M, Fuchs H. Nanomedicine - challenge and perspectives. Angew Chem Int Ed Engl. 2009;48(5):872-897.

10. Albanese A, Tang PS, Chan WC. The effect of nanoparticle size, shape, and surface chemistry on biological systems. Annu Rev Biomed Eng. 2012;14:1-16

11. Premanathan M, Karthikeyan K, Jeyasubramanian K, Manivannan G. Selective toxicity of $\mathrm{ZnO}$ nanoparticles toward Gram-positive bacteria and cancer cells by apoptosis through lipid peroxidation. Nanomedicine. 2011;7(2):184-192.

12. Debatin KM. Apoptosis pathways in cancer and cancer therapy. Cancer Immunol Immunother. 2004;53(3):153-159.

13. Seiwert TY, Salama JK, Vokes EE. The concurrent chemoradiation paradigm - general principles. Nat Clin Pract Oncol. 2007;4(2): 86-100.

14. Rasch C, Hauptmann M, Schornagel J, et al. Intra-arterial versus intravenous chemoradiation for advanced head and neck cancer: Results of a randomized phase 3 trial. Cancer. 2010;116(9):2159-2165.

15. Curran WJ, Paulus R, Langer CJ, et al. Sequential vs concurrent chemoradiation for stage III non-small cell lung cancer: randomized phase III trial RTOG 9410. J Natl Cancer Inst. 2011;103(19):1452-1460. 
16. Maas M, Beets-Tan RG, Lambregts DM, et al. Wait-and-see policy for clinical complete responders after chemoradiation for rectal cancer. J Clin Oncol. 2011;29(35):4633-4640.

17. Eifel PJ. Chemoradiotherapy in the treatment of cervical cancer. Semin Radiat Oncol. 2006;16(3):177-185.

18. Marks LB, Yorke ED, Jackson A, et al. Use of normal tissue complication probability models in the clinic. Int J Radiat Oncol. 2010; 76(3 Suppl):S10-S19.

19. Jain RK, Stylianopoulos T. Delivering nanomedicine to solid tumors. Nat Rev Clin Oncol. 2010;7(11):653-664.

20. Moghimi SM, Hunter AC, Murray JC. Long-circulating and targetspecific nanoparticles: theory to practice. Pharmacol Rev. 2001;53(2): $283-318$.

21. Hainfeld JF, Slatkin DN, Focella TM, Smilowitz HM. Gold nanoparticles: a new X-ray contrast agent. Brit J Radiol. 2006;79(939):248-253.

22. Sun S, Murray CB, Weller D, Folks L, Moser A. Monodisperse FePt nanoparticles and ferromagnetic FePt nanocrystal superlattices. Science. 2000; 287(5460):1989-1992.

23. Sun S. Recent advances in chemical synthesis, self-assembly, and applications of FePt nanoparticles. Adv Mater. 2006;18(4):393-403.

24. Hao R, Xing R, Xu Z, Hou Y, Gao S, Sun S. Synthesis, functionalization, and biomedical applications of multifunctional magnetic nanoparticles. Adv Mater. 2010;22(25):2729-2742.

25. Kitamoto Y, He JS. Chemical synthesis of FePt nanoparticles with high alternate current magnetic susceptibility for biomedical applications. Electrochim Acta. 2009;54(25):5969-5972.

26. Roca AG, Costo R, Rebolledo AF, et al. Progress in the preparation of magnetic nanoparticles for applications in biomedicine. J Phys D: Appl Phys. 2009;42(22):224002.

27. Guo S, Sun S. FePt nanoparticles assembled on graphene as enhanced catalyst for oxygen reduction reaction. $J$ Am Chem Soc. 2012;134(5):249-2495.

28. Mazumder V, Chi M, More KL, Sun S. Core/shell Pd/FePt nanoparticles as an active and durable catalyst for the oxygen reduction reaction. J Am Chem Soc. 2010;132(23):7848-7849.

29. Gao J, Liang G, Zhang B, Kuang Y, Zhang X, Xu B. FePt@CoS2 yolkshell nanocrystals as a potent agent to kill HeLa cells. J Am Chem Soc. 2007;129(5):1428-1433.

30. Yang H, Zhang J, Tian Q, et al. One-pot synthesis of amphiphilic superparamagnetic FePt NPs and magnetic resonance imaging in vitro. J Magn Mater. 2010;322(8):973-977.

31. Chen S, Wang L, Duce SL, et al. Engineered biocompatible nanoparticles for in vivo imaging applications. $J$ Am Chem Soc. 2010;132(42):15022-15029.

32. Gao J, Liang G, Cheung JS, et al. Multifunctional yolk-shell nanoparticles: a potential MRI contrast and anticancer agent. $\mathrm{J} \mathrm{Am} \mathrm{Chem} \mathrm{Soc.}$ 2008;130(35):11828-11833.

33. Chou SW, Shau YH, Wu PC, et al. In vitro and in vivo studies of FePt nanoparticles for dual modal CT/MRI molecular imaging. $J$ Am Chem Soc. 2010;132(38):13270-13278.
34. Louie A. Multimodality imaging probes: design and challenges. Chem Rev. 2010;110(5):3146-3195.

35. Sun $H$, Chen $X$, Chen $D$, et al. Influences of surface coatings and components of FePt nanoparticles on the suppression of glioma cell proliferation. Int J Nanomedicine. 2012;7:3295-3307.

36. Chou SW, Zhu CL, Neeleshwar S, Chen CL, Chen YY, Chen CC. Controlled growth and magnetic property of $\mathrm{FePt}$ nanostructure: cuboctahedron, octapod, truncated cube, and cube. Chem Mater. 2009; 21(20):4955-4961.

37. Wu Q, Cao H, Luan Q, et al. Biomolecule-assisted synthesis of watersoluble silver NPs and their biomedical applications. Inorg Chem. 2008;47(13):5882-5888.

38. Xu C, Yuan Z, Kohler N, Kim J, Chung MA, Sun S. FePt nanoparticles as an $\mathrm{Fe}$ reservoir for controlled Fe release and tumor inhibition. $J \mathrm{Am}$ Chem Soc. 2009;131(42):15346-15351.

39. Lehmann AD, Parak WJ, Zhang F, et al. Fluorescent-Magnetic hybrid nanoparticles induce a dose-dependent increase in proinflammatory response in lung cells in vitro correlated with intracellular localization. Small. 2010;6(6):753-762.

40. Liu Y, Yang K, Cheng L, et al. PEGylated FePt@, Fe2O3 core-shell magnetic nanoparticles: potential theranostic applications and in vivo toxicity studies. Nanomedicine. 2013;9(7):1077-1088.

41. Li TJ, Huang CC, Ruan PW, et al. In vivo anti-cancer efficacy of magnetite nanocrystal-based system using locoregional hyperthermia combined with 5-fluorouracil chemotherapy. Biomaterials. 2013;34(32): 7873-7883.

42. Mahmoudi M, Sant S, Wang B, Laurent S, Sen T. Superparamagnetic iron oxide nanoparticles (SPIONs): development, surface modification and applications in chemotherapy. Adv Drug Deliv Rev. 2011;63(1-2):24-46.

43. Yu MK, Park J, Jon S. Targeting strategies for multifunctional nanoparticles in cancer imaging and therapy. Theranostics. 2012;2(1):3-44.

44. Shukla R, Bansal V, Chaudhary M, Basu A, Bhonde RR, Sastry M. Biocompatibility of gold nanoparticles and their endocytotic fate inside the cellular compartment: a microscopic overview. Langmuir. 2005;21(23): 10644-10654.

45. Iversen TG, Skotland T, Sandvig K. Endocytosis and intracellular transport of nanoparticles: present knowledge and need for future studies. Nano Today. 2011;6(2):176-185.

46. Yang D, van Boom SS, Reedijk J, van Boom JH, Wang AH. Structure and isomerization of an intrastrand cisplatin-cross-linked octamer DNA duplex by NMR analysis. Biochemistry. 1995;34(39):12912-12920.

47. Takahara PM, Frederick CA, Lippard SJ. Crystal structure of the anticancer drug cisplatin bound to duplex DNA. J Am Chem Soc. 1996;118(49):12309-12321.

48. Gray LH, Conger AD, Ebert M, Hornsy S, Scott OC. The concentration of oxygen dissolved in tissues at the time of irradiation as a factor in radiotherapy. Brit J Radiol. 1953;26(312):638-648.

49. Page for X-ray mass attenuation coefficient for Pt. http://physics.nist. gov/PhysRefData/XrayMassCoef/ElemTab/z78.html
International Journal of Nanomedicine

\section{Publish your work in this journal}

The International Journal of Nanomedicine is an international, peerreviewed journal focusing on the application of nanotechnology in diagnostics, therapeutics, and drug delivery systems throughout the biomedical field. This journal is indexed on PubMed Central, MedLine, CAS, SciSearch $\AA$, Current Contents $\AA /$ Clinical Medicine,

\section{Dovepress}

Journal Citation Reports/Science Edition, EMBase, Scopus and the Elsevier Bibliographic databases. The manuscript management system is completely online and includes a very quick and fair peer-review system, which is all easy to use. Visit http://www.dovepress.com/ testimonials.php to read real quotes from published authors. 